\title{
SOME INEQUALITIES FOR $n$-TIME DIFFERENTIABLE MAPPINGS USING A MULTI-STEP KERNEL WITH APPLICATIONS
}

\section{SOFIAN OBEIDAT*}

Department of Basic Sciences, Deanship of Preparatory Year, University of Hail, Hail 2440, Saudi Arabia

${ }^{*}$ Corresponding author: obeidatsofian@gmail.com

\begin{abstract}
In this paper, we develop a new multi-step kernel and use it to establish new Ostrowski's type inequalities for $n$-time differentiable mappings, whose $n$-th derivatives satisfy convexity and quasi-convexity conditions. Applications of our findings to random variables and approximation of integrals are given.
\end{abstract}

\section{INTRODUCTION}

The classical Ostrowski inequality $\left(1938\right.$, see [8]) is given as follows: if $x \in\left[x_{1}, x_{2}\right]$ then

$$
\left|g(x)-\frac{1}{x_{2}-x_{1}} \int_{x_{1}}^{x_{2}} g(t) d t\right| \leq\left[\frac{1}{4}+\frac{\left(x-\frac{x_{1}+x_{2}}{2}\right)^{2}}{\left(x_{2}-x_{1}\right)^{2}}\right]\left(x_{2}-x_{1}\right)\left\|g^{\prime}\right\|_{\infty},
$$

where $g$ is a differentiable function defined on a finite interval $\left[x_{1}, x_{2}\right]$, whose derivative is integrable and bounded over $\left[x_{1}, x_{2}\right]$. The constant $1 / 4$ is the best possible. When $x=\frac{x_{1}+x_{2}}{2}$, Inequality 1.1 reduces to the midpoint version

$$
\left|g\left(\frac{x_{1}+x_{2}}{2}\right)-\frac{1}{x_{2}-x_{1}} \int_{x_{1}}^{x_{2}} g(t) d t\right| \leq \frac{\left(x_{2}-x_{1}\right)}{4}\left\|g^{\prime}\right\|_{\infty} .
$$

The importance of Ostrowski's type inequalities is due to their applications in different aspects. For generalizations and variants of Ostrowski's type inequalities, we refer the reader to [2] and [4].

Received 2019-10-26; accepted 2019-11-22; published 2020-01-02.

2010 Mathematics Subject Classification. Primary 26D15, 26D20; Secondary 26D99.

Key words and phrases. convex functions; Hölder inequality; quasi-convex function; Ostrowski inequality.

(c)2020 Authors retain the copyrights of their papers, and all open access articles are distributed under the terms of the Creative Commons Attribution License. 
Recently, several authors have derived Ostrowski's type inequalities for $n$-time differentiable mappings whose $n$-th derivatives satisfy different types of convexity conditions, see for example [?], [3], [5], [7], [10] and $[12]$.

In particular, Ozdemir and Yildiz, in [9], obtained the following three theorems for $n$-time differentiable mappings .

Theorem 1.1. [9] Suppose that $n$ is a positive integer, $g: J \subseteq \mathbb{R} \rightarrow \mathbb{R}$ is $n$-time differentiable mapping on $J^{\circ}$ and $x_{1}, x_{2} \in J^{\circ}$ with $x_{1}<x_{2}$. If $g^{(n)} \in L_{1}\left(\left[x_{1}, x_{2}\right]\right)$ and $\left|g^{(n)}\right|$ is convex on $\left[x_{1}, x_{2}\right]$, then

$$
\begin{aligned}
& \left|\int_{x_{1}}^{x_{2}} g(x) d x-\sum_{k=0}^{n-1} \frac{\left(1+(-1)^{k}\right)\left(x_{2}-x_{1}\right)^{k+1}}{2^{k+1}(k+1) !} g^{(k)}\left(\frac{x_{1}+x_{2}}{2}\right)\right| \\
\leq & \frac{\left(x_{2}-x_{1}\right)^{n+1}}{2^{n}(n+1) !}\left[\frac{\left|g^{(n)}\left(x_{1}\right)\right|+\left|g^{(n)}\left(x_{2}\right)\right|}{2}\right] .
\end{aligned}
$$

Theorem 1.2. [9] Suppose that $n$ is a positive integer, $g: J \subseteq \mathbb{R} \rightarrow \mathbb{R}$ is $n$-time differentiable mapping on $J^{\circ}$ and $x_{1}, x_{2} \in J^{\circ}$ with $x_{1}<x_{2}$. If $g^{(n)} \in L_{1}\left(\left[x_{1}, x_{2}\right]\right)$ and $\left|g^{(n)}\right|^{q}$ is convex on $\left[x_{1}, x_{2}\right]$, where $q>1$, then

$$
\begin{aligned}
& \left|\int_{x_{1}}^{x_{2}} g(x) d x-\sum_{k=0}^{n-1} \frac{\left(1+(-1)^{k}\right)\left(x_{2}-x_{1}\right)^{k+1}}{2^{k+1}(k+1) !} g^{(k)}\left(\frac{x_{1}+x_{2}}{2}\right)\right| \\
& \leq \frac{\left(x_{2}-x_{1}\right)^{n+1}}{2^{n+1} n !}\left(\frac{1}{1+p n}\right)^{\frac{1}{p}}\left[\left(\frac{\left|g^{(n)}\left(x_{1}\right)\right|^{q}+3\left|g^{(n)}\left(x_{2}\right)\right|^{q}}{4}\right)^{\frac{1}{q}}\right. \\
& \left.\quad+\frac{3\left|g^{(n)}\left(x_{1}\right)\right|^{q}+\left|g^{(n)}\left(x_{2}\right)\right|^{q}}{4}\right],
\end{aligned}
$$

where $\frac{1}{p}+\frac{1}{q}=1$.

Theorem 1.3. [9] Suppose that $n$ is a positive integer, $g: J \subseteq \mathbb{R} \rightarrow \mathbb{R}$ is $n$-time differentiable mapping on $J^{\circ}$ and $x_{1}, x_{2} \in J^{\circ}$ with $x_{1}<x_{2}$. If $g^{(n)} \in L_{1}\left(\left[x_{1}, x_{2}\right]\right)$ and $\left|g^{(n)}\right|^{q}$ is convex on $\left[x_{1}, x_{2}\right]$, where $q \geq 1$, then

$$
\begin{aligned}
& \left|\int_{x_{1}}^{x_{2}} g(x) d x-\sum_{k=0}^{n-1} \frac{\left(1+(-1)^{k}\right)\left(x_{2}-x_{1}\right)^{k+1}}{2^{k+1}(k+1) !} g^{(k)}\left(\frac{x_{1}+x_{2}}{2}\right)\right| \\
& \leq \frac{\left(x_{2}-x_{1}\right)^{n+1}}{2^{n+1}(n+1) !}\left[\left(\frac{n+1}{2 n+4}\left|g^{(n)}\left(x_{1}\right)\right|^{q}+\frac{n+3}{2 n+4}\left|g^{(n)}\left(x_{2}\right)\right|^{q}\right)^{\frac{1}{q}}\right. \\
& \left.\quad+\left(\frac{n+3}{2 n+4}\left|g^{(n)}\left(x_{1}\right)\right|^{q}+\frac{n+1}{2 n+4}\left|g^{(n)}\left(x_{2}\right)\right|^{q}\right)^{\frac{1}{q}}\right] .
\end{aligned}
$$

In this paper, we generalize the inequalities obtained by Ozdemir and Yildiz in [9] for convex and quasi convex functions using a multi-step kernel. Then we introduce some applications of our findings to random variables and approximation of integrals. Throught this paper, $\mathbb{R}$ denotes the set of all real numbers and $J \subset \mathbb{R}$ denotes an interval. The concepts of convex and quasi convex functions, which are well known in the literature, are given as in the following two definitions. 
Definition 1.1. [6] A function $g: J \subset \mathbb{R} \rightarrow \mathbb{R}$ is said to be convex if the inequality

$$
g\left(t x_{1}+(1-t) x_{2}\right) \leq t g\left(x_{1}\right)+(1-t) g\left(x_{2}\right)
$$

holds for all $x_{1}, x_{2} \in J$ and $t \in[0,1]$.

Definition 1.2. [11] A function $g: J \subset \mathbb{R} \rightarrow \mathbb{R}$ is said to be quasi convex if the inequality

$$
g\left(t x_{1}+(1-t) x_{2}\right) \leq \max \left\{g\left(x_{1}\right), g\left(x_{2}\right)\right\}
$$

holds for all $x_{1}, x_{2} \in J$ and $t \in[0,1]$.

\section{Main Results}

We start this section with the following two Lemmas.

Lemma 2.1. Suppose that $n$ and $m$ are positive integers, $g: J \subseteq \mathbb{R} \rightarrow \mathbb{R}$ is $n$-time differentiable mapping on $J^{\circ}$ and $x_{1}, x_{2} \in J^{\circ}$ with $x_{1}<x_{2}$. Suppose that $g^{(n)} \in L_{1}\left(\left[x_{1}, x_{2}\right]\right)$. Then for every $s_{1}, s_{2} \in[0,1]$ the identity

$$
\begin{aligned}
& \int_{s_{1}}^{s_{2}} g\left(x_{2}+t\left(x_{1}-x_{2}\right)\right) d t \\
= & \sum_{k=0}^{n-1} \frac{\left[\left(s_{2}-r\right)^{k+1} g^{(k)}\left(x_{2}+s_{2}\left(x_{1}-x_{2}\right)\right)-\left(s_{1}-r\right)^{k+1} g^{(k)}\left(x_{2}+s_{1}\left(x_{1}-x_{2}\right)\right)\right]}{\left(x_{2}-x_{1}\right)^{-k}(k+1) !} \\
& +\frac{\left(x_{2}-x_{1}\right)^{n}}{n !} \int_{s_{1}}^{s_{2}}(t-r)^{n} g^{(n)}\left(x_{2}+t\left(x_{1}-x_{2}\right)\right) d t,
\end{aligned}
$$

holds for each $r \in \mathbb{R}$.

Proof. Using integration by parts repeatedly, we get that

$$
\begin{aligned}
& \frac{1}{n !} \int_{s_{1}}^{s_{2}}(t-r)^{n} g^{(n)}\left(x_{2}+t\left(x_{1}-x_{2}\right)\right) d t \\
= & -\left.\sum_{k=0}^{n-1} \frac{(t-r)^{k+1} g^{(k)}\left(x_{2}+t\left(x_{1}-x_{2}\right)\right)}{\left(x_{2}-x_{1}\right)^{n-k}(k+1) !}\right|_{s_{1}} ^{s_{2}} \\
+ & \frac{1}{\left(x_{2}-x_{1}\right)^{n}} \int_{s_{1}}^{s_{2}} g\left(x_{2}+t\left(x_{1}-x_{2}\right)\right) d t .
\end{aligned}
$$

Multiplying (2.2) by $\left(x_{2}-x_{1}\right)^{n}$, and substituting the upper and lower integral bounds, the result follows. 
Lemma 2.2. Suppose that $n$ and $m$ are positive integers, $g: J \subseteq \mathbb{R} \rightarrow \mathbb{R}$ is $n$-time differentiable mapping on $J^{\circ}$ and $x_{1}, x_{2} \in J^{\circ}$ with $x_{1}<x_{2}$. If $g^{(n)} \in L_{1}\left(\left[x_{1}, x_{2}\right]\right)$ then

$$
\begin{aligned}
& \int_{x_{1}}^{x_{2}} g(x) d x \\
= & \sum_{l=1}^{2^{m-1}} \sum_{k=0}^{n-1} \frac{\left[1+(-1)^{k}\right] 2^{-m(k+1)}}{\left(x_{2}-x_{1}\right)^{-k-1}(k+1) !} g^{(k)}\left(\frac{(2 l-1) x_{1}+\left(2^{m}-2 l+1\right) x_{2}}{2^{m}}\right) \\
& +\left(x_{2}-x_{1}\right)^{n+1} \int_{0}^{1} P_{n, m}(t) g^{(n)}\left(x_{2}+t\left(x_{1}-x_{2}\right)\right) d t
\end{aligned}
$$

where

$$
P_{n, m}(t)=\frac{1}{n !}\left\{\begin{array}{cc}
t^{n}, & \text { if } t \in\left[0, \frac{1}{2^{m}}\right] \\
\left(t-2^{1-m} l\right)^{n}, & \text { if } t \in\left(\frac{2 l-1}{2^{m}}, \frac{2 l+1}{2^{m}}\right], l=1,2, \cdots, 2^{m-1}-1 \\
(t-1)^{n}, & \text { if } t \in\left(1-\frac{1}{2^{m}}, 1\right]
\end{array}\right.
$$

Proof. Using Lemma 2.1, we have

$$
\begin{aligned}
& \int_{0}^{\frac{1}{2^{m}}} g\left(x_{2}+t\left(x_{1}-x_{2}\right)\right) d t \\
= & \sum_{k=0}^{n-1} \frac{\left[\left(\frac{1}{2^{m}}\right)^{k+1} g^{(k)}\left(\frac{x_{1}+\left(2^{m}-1\right) x_{2}}{2^{m}}\right)\right]}{\left(x_{2}-x_{1}\right)^{-k}(k+1) !} \\
& +\frac{\left(x_{2}-x_{1}\right)^{n}}{n !} \int_{0}^{\frac{1}{2^{m}}} P_{n, m}(t) g^{(n)}\left(x_{2}+t\left(x_{1}-x_{2}\right)\right) d t, \\
& \int_{\left(1-\frac{1}{2^{m}}\right)}^{1} g\left(x_{2}+t\left(x_{1}-x_{2}\right)\right) d t \\
= & \sum_{k=0}^{n-1} \frac{\left(\frac{-1}{2^{m}}\right)^{k+1} g^{(k)}\left(\frac{\left(2^{m}-1\right) x_{1}+x_{2}}{2^{m}}\right)}{\left(x_{2}-x_{1}\right)^{-k}(k+1) !} \\
& +\frac{\left(x_{2}-x_{1}\right)^{n}}{n !} \int_{\left(1-\frac{1}{2^{m}}\right)}^{1} P_{n, m}(t) g^{(n)}\left(x_{2}+t\left(x_{1}-x_{2}\right)\right) d t
\end{aligned}
$$

and

$$
\begin{aligned}
& \int_{\frac{2 l-1}{2^{m}}}^{\frac{2 l+1}{2^{m}}} g\left(x_{2}+t\left(x_{1}-x_{2}\right)\right) d t \\
= & \sum_{k=0}^{n-1} \frac{\left[g^{(k)}\left(\frac{(2 l+1) x_{1}+\left(2^{m}-2 l-1\right) x_{2}}{2^{m}}\right)+(-1)^{k} g^{(k)}\left(\frac{(2 l-1) x_{1}+\left(2^{m}-2 l+1\right) x_{2}}{2^{m}}\right)\right]}{2^{m(k+1)}\left(x_{2}-x_{1}\right)^{-k}(k+1) !} \\
& +\frac{\left(x_{2}-x_{1}\right)^{n}}{n !} \int_{\frac{2 l-1}{2^{m}}}^{\frac{2 l+1}{2^{m}}} P_{n, m}(t) g^{(n)}\left(x_{2}+t\left(x_{1}-x_{2}\right)\right) d t
\end{aligned}
$$

for $l=1,2, \cdots, 2^{m-1}-1$. Combining (2.4), (2.5) and (2.6), the result follows. 
Theorem 2.1. Suppose that $n$ and $m$ are positive integers, $g: J \subseteq \mathbb{R} \rightarrow \mathbb{R}$ is $n$-time differentiable mapping on $J^{\circ}$ and $x_{1}, x_{2} \in J^{\circ}$ with $x_{1}<x_{2}$. If $g^{(n)} \in L_{1}\left(\left[x_{1}, x_{2}\right]\right)$ and $\left|g^{(n)}\right|$ is convex on $\left[x_{1}, x_{2}\right]$, then

$$
\begin{aligned}
& \mid \int_{x_{1}}^{x_{2}} g(x) d x-\sum_{l=1}^{2^{m-1}} \sum_{k=0}^{n-1}\left[\frac{\left(1+(-1)^{k}\right) 2^{-m(k+1)}}{\left(x_{2}-x_{1}\right)^{-k-1}(k+1) !}\right. \\
& \left.\quad \times g^{(k)}\left(\frac{(2 l-1) x_{1}+\left(2^{m}-2 l+1\right) x_{2}}{2^{m}}\right)\right] \mid \\
& \leq \frac{\left(x_{2}-x_{1}\right)^{n+1}}{2^{m n}(n+1) !}\left[\frac{\left|g^{(n)}\left(x_{1}\right)\right|+\left|g^{(n)}\left(x_{2}\right)\right|}{2}\right] .
\end{aligned}
$$

Proof. Using convexity of $\left|g^{(n)}\right|$ on $\left[x_{1}, x_{2}\right]$, we get that

$$
\begin{aligned}
& \left|\int_{0}^{1} P_{n, m}(t) g^{(n)}\left(x_{2}+t\left(x_{1}-x_{2}\right)\right) d t\right| \\
\leq & \frac{1}{n !} \int_{0}^{\frac{1}{2^{m}}} t^{n}\left[(1-t)\left|g^{(n)}\left(x_{2}\right)\right|+t\left|g^{(n)}\left(x_{1}\right)\right|\right] d t \\
& +\frac{1}{n !} \sum_{l=1}^{2^{m-1}-1} \int_{\frac{2 l-1}{2^{m}}}^{\frac{2 l+1}{2^{m}}}\left|t-\frac{l}{2^{m-1}}\right|^{n}\left[(1-t)\left|g^{(n)}\left(x_{2}\right)\right|+t\left|g^{(n)}\left(x_{1}\right)\right|\right] d t \\
& +\frac{1}{n !} \int_{1-\frac{1}{2^{m}}}^{1}(1-t)^{n}\left[(1-t)\left|g^{(n)}\left(x_{2}\right)\right|+t\left|g^{(n)}\left(x_{1}\right)\right|\right] d t \\
= & \frac{1}{2^{m n}(n+1) !}\left[\frac{\left|g^{(n)}\left(x_{1}\right)\right|+\left|g^{(n)}\left(x_{2}\right)\right|}{2}\right] .
\end{aligned}
$$

Using Identity 2.3 and Inequality 2.8 , the result follows.

Remark 2.1. In Theorem 2.1,

(1) If $m=1$, Inequality 2.7 reduces to Inequality 1.2.

(2) If $m=2$, Inequality 2.7 reduces to

$$
\begin{aligned}
& \mid \int_{x_{1}}^{x_{2}} g(x) d x-\sum_{k=0}^{n-1} \frac{\left(1+(-1)^{k}\right) 2^{-2(k+1)}}{\left(x_{2}-x_{1}\right)^{-k-1}(k+1) !}\left[g^{(k)}\left(\frac{3 x_{1}+x_{2}}{4}\right)\right. \\
& \left.\quad+g^{(k)}\left(\frac{x_{1}+3 x_{2}}{4}\right)\right] \mid \\
& \leq \frac{\left(x_{2}-x_{1}\right)^{n+1}}{2^{2 n}(n+1) !}\left[\frac{\left|g^{(n)}\left(x_{1}\right)\right|+\left|g^{(n)}\left(x_{2}\right)\right|}{2}\right] .
\end{aligned}
$$


(3) If $m=3$, Inequality 2.7 reduces to

$$
\begin{aligned}
& \mid \int_{x_{1}}^{x_{2}} g(x) d x-\sum_{k=0}^{n-1} \frac{\left(1+(-1)^{k}\right) 2^{-3(k+1)}}{\left(x_{2}-x_{1}\right)^{-k-1}(k+1) !}\left[g^{(k)}\left(\frac{7 x_{1}+x_{2}}{8}\right)\right. \\
& \left.\quad+g^{(k)}\left(\frac{5 x_{1}+3 x_{2}}{8}\right)+g^{(k)}\left(\frac{3 x_{1}+5 x_{2}}{8}\right)+g^{(k)}\left(\frac{x_{1}+7 x_{2}}{8}\right)\right] \mid \\
& \leq \frac{\left(x_{2}-x_{1}\right)^{n+1}}{2^{3 n}(n+1) !}\left[\frac{\left|g^{(n)}\left(x_{1}\right)\right|+\left|g^{(n)}\left(x_{2}\right)\right|}{2}\right] .
\end{aligned}
$$

Theorem 2.2. Suppose that $n$ and $m$ are positive integers, $g: J \subseteq \mathbb{R} \rightarrow \mathbb{R}$ is $n$-time differentiable mapping on $J^{\circ}$ and $x_{1}, x_{2} \in J^{\circ}$ with $x_{1}<x_{2}$. If $g^{(n)} \in L_{1}\left(\left[x_{1}, x_{2}\right]\right)$ and $\left|g^{(n)}\right|^{q}$ is convex on $\left[x_{1}, x_{2}\right]$, where $q>1$, then

$$
\begin{aligned}
& \mid \int_{x_{1}}^{x_{2}} g(x) d x-\sum_{l=1}^{2^{m-1}} \sum_{k=0}^{n-1}\left[\frac{\left(1+(-1)^{k}\right) 2^{-m(k+1)}}{\left(x_{2}-x_{1}\right)^{-k-1}(k+1) !}\right. \\
& \left.\times g^{(k)}\left(\frac{(2 l-1) x_{1}+\left(2^{m}-2 l+1\right) x_{2}}{2^{m}}\right)\right] \mid \\
& \leq \quad \frac{\left(x_{2}-x_{1}\right)^{n+1}}{2^{m(n+1)} n !}\left(\frac{1}{1+p n}\right)^{\frac{1}{p}} \\
& \times\left[\left(\frac{1}{2^{m+1}}\left|g^{(n)}\left(x_{1}\right)\right|^{q}+\left(1-\frac{1}{2^{m+1}}\right)\left|g^{(n)}\left(x_{2}\right)\right|^{q}\right)^{\frac{1}{q}}\right. \\
& +\left(\left(1-\frac{1}{2^{m+1}}\right)\left|g^{(n)}\left(x_{1}\right)\right|^{q}+\frac{1}{2^{m+1}}\left|g^{(n)}\left(x_{2}\right)\right|^{q}\right)^{\frac{1}{q}} \\
& \left.+2 \sum_{l=1}^{2^{m-1}-1}\left(\frac{l}{2^{m-1}}\left|g^{(n)}\left(x_{1}\right)\right|^{q}+\left(1-\frac{l}{2^{m-1}}\right)\left|g^{(n)}\left(x_{2}\right)\right|^{q}\right)^{\frac{1}{q}}\right],
\end{aligned}
$$

where $\frac{1}{p}+\frac{1}{q}=1$

Proof. Using Holder's inequality, we get that

$$
\begin{aligned}
& \left|\int_{0}^{1} P_{n, m}(t) g^{(n)}\left(x_{2}+t\left(x_{1}-x_{2}\right)\right) d t\right| \\
& \leq \frac{1}{n !}\left(\int_{0}^{\frac{1}{2^{m}}} t^{p n} d t\right)^{\frac{1}{p}}\left(\int_{0}^{\frac{1}{2^{m}}}\left|g^{(n)}\left(x_{2}+t\left(x_{1}-x_{2}\right)\right)\right|^{q} d t\right)^{\frac{1}{q}} \\
& +\frac{1}{n !} \sum_{l=1}^{2^{m-1}-1}\left(\int_{\frac{2 l-1}{2^{m}}}^{\frac{2 l+1}{2^{m}}}\left|t-\frac{l}{2^{m-1}}\right|^{p n} d t\right)^{\frac{1}{p}}\left(\int_{\frac{2 l-1}{2^{m}}}^{\frac{2 l+1}{2^{m}}}\left|g^{(n)}\left(x_{2}+t\left(x_{1}-x_{2}\right)\right)\right|^{q} d t\right)^{\frac{1}{q}} \\
& +\frac{1}{n !}\left(\int_{1-\frac{1}{2^{m}}}^{1}(1-t)^{p n} d t\right)^{\frac{1}{p}}\left(\int_{1-\frac{1}{2^{m}}}^{1}\left|g^{(n)}\left(x_{2}+t\left(x_{1}-x_{2}\right)\right)\right|^{q} d t\right)^{\frac{1}{q}} .
\end{aligned}
$$


Using convexity of $\left|g^{(n)}\right|^{q}$ on $\left[x_{1}, x_{2}\right]$, we find that

$$
\begin{aligned}
& \left|\int_{0}^{1} P_{n, m}(t) g^{(n)}\left(x_{2}+t\left(x_{1}-x_{2}\right)\right) d t\right| \\
\leq & \frac{1}{2^{m(n+1)} n !}\left(\frac{1}{1+p n}\right)^{\frac{1}{p}} \\
& \times\left[\left(\frac{1}{2^{m+1}}\left|g^{(n)}\left(x_{1}\right)\right|^{q}+\left(1-\frac{1}{2^{m+1}}\right)\left|g^{(n)}\left(x_{2}\right)\right|^{q}\right)^{\frac{1}{q}}\right. \\
& +\left(\left(1-\frac{1}{2^{m+1}}\right)\left|g^{(n)}\left(x_{1}\right)\right|^{q}+\frac{1}{2^{m+1}}\left|g^{(n)}\left(x_{1}\right)\right|^{q}\right)^{\frac{1}{q}} \\
& \left.+2 \sum_{l=1}^{2^{m-1}-1}\left(\frac{l}{2^{m-1}}\left|g^{(n)}\left(x_{1}\right)\right|^{q}+\left(1-\frac{l}{2^{m-1}}\right)\left|g^{(n)}\left(x_{2}\right)\right|^{q}\right)^{\frac{1}{q}}\right] .
\end{aligned}
$$

Using Identity 2.3 and Inequality 2.13 , the result follows.

Remark 2.2. In Theorem 2.2,

(1) If $m=1$, Inequality 2.11 reduces to Inequality 1.3.

(2) If $m=2$, Inequality 2.11 reduces to

$$
\begin{aligned}
& \mid \int_{x_{1}}^{x_{2}} g(x) d x-\sum_{k=0}^{n-1}\left[\frac { ( 1 + ( - 1 ) ^ { k } ) 2 ^ { - 2 ( k + 1 ) } } { ( x _ { 2 } - x _ { 1 } ) ^ { - k - 1 } ( k + 1 ) ! } \left(g^{(k)}\left(\frac{3 x_{1}+x_{2}}{4}\right)\right.\right. \\
& \left.\left.+g^{(k)}\left(\frac{x_{1}+3 x_{2}}{4}\right)\right)\right] \mid \\
& \leq \frac{\left(x_{2}-x_{1}\right)^{n+1}}{2^{2(n+1)} n !}\left(\frac{1}{1+p n}\right)^{\frac{1}{p}}\left[2\left(\frac{\left|g^{(n)}\left(x_{1}\right)\right|^{q}+\left|g^{(n)}\left(x_{2}\right)\right|^{q}}{2}\right)^{\frac{1}{q}}\right. \\
& \left.\quad+\left(\frac{7\left|g^{(n)}\left(x_{1}\right)\right|^{q}+\left|g^{(n)}\left(x_{2}\right)\right|^{q}}{8}\right)^{\frac{1}{q}}+\left(\frac{\left|g^{(n)}\left(x_{1}\right)\right|^{q}+7\left|g^{(n)}\left(x_{2}\right)\right|^{q}}{8}\right)^{\frac{1}{q}}\right] .
\end{aligned}
$$

Theorem 2.3. Suppose that $n$ and $m$ are positive integers, $g: J \subseteq \mathbb{R} \rightarrow \mathbb{R}$ is $n$-time differentiable mapping on $J^{\circ}$ and $x_{1}, x_{2} \in J^{\circ}$ with $x_{1}<x_{2}$. If $g^{(n)} \in L_{1}\left(\left[x_{1}, x_{2}\right]\right)$ and $\left|g^{(n)}\right|^{q}$ is convex on $\left[x_{1}, x_{2}\right]$, where $q \geq 1$, then

$$
\begin{aligned}
& \mid \int_{x_{1}}^{x_{2}} g(x) d x-\sum_{l=1}^{2^{m-1}} \sum_{k=0}^{n-1}\left[\frac{\left(1+(-1)^{k}\right) 2^{-m(k+1)}}{\left(x_{2}-x_{1}\right)^{-k-1}(k+1) !}\right. \\
& \left.\times g^{(k)}\left(\frac{(2 l-1) x_{1}+\left(2^{m}-2 l+1\right) x_{2}}{2^{m}}\right)\right] \mid \\
& \leq \frac{\left(x_{2}-x_{1}\right)^{n+1}}{2^{m(n+1)}(n+1) !} \\
& \quad \times\left[\left(\frac{2^{-m}(n+1)}{(n+2)}\left|g^{(n)}\left(x_{1}\right)\right|^{q}+\left(1-\frac{2^{-m}(n+1)}{(n+2)}\right)\left|g^{(n)}\left(x_{2}\right)\right|^{q}\right)^{\frac{1}{q}}\right.
\end{aligned}
$$




$$
\begin{aligned}
& +2 \sum_{l=1}^{2^{m-1}-1}\left(2^{1-m}\left|g^{(n)}\left(x_{1}\right)\right|^{q}+\left(1-2^{1-m} l\right)\left|g^{(n)}\left(x_{2}\right)\right|^{q}\right)^{\frac{1}{q}} \\
& \left.+\left(\left(1-\frac{2^{-m}(n+1)}{(n+2)}\right)\left|g^{(n)}\left(x_{1}\right)\right|^{q}+\frac{2^{-m}(n+1)}{(n+2)}\left|g^{(n)}\left(x_{2}\right)\right|^{q}\right)^{\frac{1}{q}}\right] .
\end{aligned}
$$

Proof. Using convexity of $\left|g^{(n)}\right|^{q}$ on $\left[x_{1}, x_{2}\right]$, we get that

$$
\begin{aligned}
& \left|\int_{0}^{1} P_{n, m}(t) g^{(n)}\left(x_{2}+t\left(x_{1}-x_{2}\right)\right) d t\right| \\
& \leq \frac{1}{n !}\left(\int_{0}^{\frac{1}{2^{m}}} t^{n} d t\right)^{\frac{1}{p}}\left(\int_{0}^{\frac{1}{2^{m}}} t^{n}\left|g^{(n)}\left(x_{2}+t\left(x_{1}-x_{2}\right)\right)\right|^{q} d t\right)^{\frac{1}{q}} \\
& +\frac{1}{n !}\left(\int_{1-\frac{1}{2^{m}}}^{1}(1-t)^{n} d t\right)^{\frac{1}{p}}\left(\int_{1-\frac{1}{2^{m}}}^{1}(1-t)^{n}\left|g^{(n)}\left(x_{2}+t\left(x_{1}-x_{2}\right)\right)\right|^{q} d t\right)^{\frac{1}{q}} \\
& +\frac{1}{n !} \sum_{l=1}^{2^{m-1}-1}\left[\left(\int_{\frac{2 l-1}{2^{m}}}^{\frac{2 l+1}{2^{m}}}\left|t-\frac{l}{2^{m-1}}\right|^{n} d t\right)^{\frac{1}{p}}\right. \\
& \left.\times\left(\int_{\frac{2 l-1}{2^{m}}}^{\frac{2 l+1}{2^{m}}}\left|t-\frac{l}{2^{m-1}}\right|^{n}\left|g^{(n)}\left(x_{2}+t\left(x_{1}-x_{2}\right)\right)\right|^{q} d t\right)^{\frac{1}{q}}\right] \\
& \leq \frac{2^{-m(n+1)}}{(n+1) !}\left(\frac{2^{-m}(n+1)}{(n+2)}\left|g^{(n)}\left(x_{1}\right)\right|^{q}+\left(1-\frac{2^{-m}(n+1)}{(n+2)}\right)\left|g^{(n)}\left(x_{2}\right)\right|^{q}\right)^{\frac{1}{q}} \\
& +\frac{2^{-m(n+1)}}{(n+1) !}\left(\left(1-\frac{2^{-m}(n+1)}{(n+2)}\right)\left|g^{(n)}\left(x_{1}\right)\right|^{q}+\frac{2^{-m}(n+1)}{(n+2)}\left|g^{(n)}\left(x_{2}\right)\right|^{q}\right)^{\frac{1}{q}} \\
& +\frac{2^{-m(n+1)+1}}{(n+1) !} \sum_{l=1}^{2^{m-1}-1}\left(2^{1-m}\left|g^{(n)}\left(x_{1}\right)\right|^{q}+\left(1-2^{1-m} l\right)\left|g^{(n)}\left(x_{2}\right)\right|^{q}\right)^{\frac{1}{q}} .
\end{aligned}
$$

Using (2.3) and (2.16), the result follows.

\section{Remark 2.3. In Theorem 2.3,}

(1) If $m=1$, Inequality 2.15 reduces to Inequality 1.4.

(2) If $m=2$, Inequality 2.15 reduces to

$$
\begin{aligned}
& \mid \int_{x_{1}}^{x_{2}} g(x) d x-\sum_{k=0}^{n-1}\left[\frac { ( 1 + ( - 1 ) ^ { k } ) 2 ^ { - 2 ( k + 1 ) } } { ( x _ { 2 } - x _ { 1 } ) ^ { - k - 1 } ( k + 1 ) ! } \left(g^{(k)}\left(\frac{3 x_{1}+x_{2}}{4}\right)\right.\right. \\
& \left.\left.\quad+g^{(k)}\left(\frac{x_{1}+3 x_{2}}{4}\right)\right)\right] \mid \\
& \leq \quad \frac{\left(x_{2}-x_{1}\right)^{n+1}}{2^{2(n+1)}(n+1) !}\left(\left(\frac{n+1}{4 n+8}\right)\left|g^{(n)}\left(x_{1}\right)\right|^{q}+\left(\frac{3 n+7}{4 n+8}\right)\left|g^{(n)}\left(x_{2}\right)\right|^{q}\right)^{\frac{1}{q}} \\
& \quad+\frac{2\left(x_{2}-x_{1}\right)^{n+1}}{2^{2(n+1)}(n+1) !}\left(\frac{\left|g^{(n)}\left(x_{1}\right)\right|^{q}+\left|g^{(n)}\left(x_{2}\right)\right|^{q}}{2}\right)^{\frac{1}{q}} \\
& \quad+\frac{\left(x_{2}-x_{1}\right)^{n+1}}{2^{2(n+1)}(n+1) !}\left(\left(\frac{3 n+7}{4 n+8}\right)\left|g^{(n)}\left(x_{1}\right)\right|^{q}+\left(\frac{n+1}{4 n+8}\right)\left|g^{(n)}\left(x_{2}\right)\right|^{q}\right)^{\frac{1}{q}} .
\end{aligned}
$$


Theorem 2.4. Suppose that $n$ and $m$ are positive integers, $g: J \subseteq \mathbb{R} \rightarrow \mathbb{R}$ is $n$-time differentiable mapping on $J^{\circ}$ and $x_{1}, x_{2} \in J^{\circ}$ with $x_{1}<x_{2}$. If $g^{(n)} \in L_{1}\left(\left[x_{1}, x_{2}\right]\right)$ and $\left|g^{(n)}\right|$ is quasi-convex on $\left[x_{1}, x_{2}\right]$, then

$$
\begin{aligned}
& \mid \int_{x_{1}}^{x_{2}} g(x) d x-\sum_{l=1}^{2^{m-1}} \sum_{k=0}^{n-1}\left[\frac{\left(1+(-1)^{k}\right) 2^{-m(k+1)}}{\left(x_{2}-x_{1}\right)^{-k-1}(k+1) !}\right. \\
& \left.\quad \times g^{(k)}\left(\frac{(2 l-1) x_{1}+\left(2^{m}-2 l+1\right) x_{2}}{2^{m}}\right)\right] \mid \\
& \leq \frac{\left(x_{2}-x_{1}\right)^{n+1}}{2^{m n}(n+1) !} \max \left\{\left|g^{(n)}\left(x_{1}\right)\right|,\left|g^{(n)}\left(x_{2}\right)\right|\right\} .
\end{aligned}
$$

Proof. Using quasi-convexity of $\left|g^{(n)}\right|$ on $\left[x_{1}, x_{2}\right]$, we get that

$$
\begin{aligned}
& \left|\int_{0}^{1} P_{n, m}(t) g^{(n)}\left(x_{2}+t\left(x_{1}-x_{2}\right)\right) d t\right| \\
\leq & \frac{\max \left\{\left|g^{(n)}\left(x_{1}\right)\right|,\left|g^{(n)}\left(x_{2}\right)\right|\right\}}{n !}\left[\int_{0}^{\frac{1}{2^{m}}} t^{n} d t\right. \\
& \left.+\sum_{l=1}^{2^{m-1}-1} \int_{\frac{2 l-1}{2^{m}}}^{\frac{2 l+1}{2^{m}}}\left|t-\frac{l}{2^{m-1}}\right|^{n} d t+\int_{1-\frac{1}{2^{m}}}^{1}(1-t)^{n} d t\right] \\
= & \frac{1}{2^{m n}(n+1) !} \max \left\{\left|g^{(n)}\left(x_{1}\right)\right|,\left|g^{(n)}\left(x_{2}\right)\right|\right\} .
\end{aligned}
$$

Using Identity 2.3 and Inequality 2.19 , the result follows.

Theorem 2.5. Suppose that $n$ and $m$ are positive integers, $g: J \subseteq \mathbb{R} \rightarrow \mathbb{R}$ is $n$-time differentiable mapping on $J^{\circ}$ and $x_{1}, x_{2} \in J^{\circ}$ with $x_{1}<x_{2}$. If $g^{(n)} \in L_{1}\left(\left[x_{1}, x_{2}\right]\right)$ and $\left|g^{(n)}\right|^{q}$ is quasi-convex on $\left[x_{1}, x_{2}\right]$, where $q>1$, then

$$
\begin{aligned}
& \mid \int_{x_{1}}^{x_{2}} g(x) d x-\sum_{l=1}^{2^{m-1}} \sum_{k=0}^{n-1}\left[\frac{\left(1+(-1)^{k}\right) 2^{-m(k+1)}}{\left(x_{2}-x_{1}\right)^{-k-1}(k+1) !}\right. \\
& \left.\quad \times g^{(k)}\left(\frac{(2 l-1) x_{1}+\left(2^{m}-2 l+1\right) x_{2}}{2^{m}}\right)\right] \mid \\
& \leq \frac{\left(x_{2}-x_{1}\right)^{n+1}}{2^{m n} n !}\left(\frac{1}{1+p n}\right)^{\frac{1}{p}}\left(\max \left\{\left|g^{(n)}\left(x_{1}\right)\right|^{q},\left|g^{(n)}\left(x_{2}\right)\right|^{q}\right\}\right)^{\frac{1}{q}},
\end{aligned}
$$

where $\frac{1}{p}+\frac{1}{q}=1$. 
Proof. Using Holder's inequality and quasi-convexity of $\left|g^{(n)}\right|^{q}$ on $\left[x_{1}, x_{2}\right]$, we get that

$$
\begin{aligned}
& \left|\int_{0}^{1} P_{n, m}(t) g^{(n)}\left(x_{2}+t\left(x_{1}-x_{2}\right)\right) d t\right| \\
\leq & \frac{2^{-m(n+1)+1}}{n !}\left(\frac{1}{1+p n}\right)^{\frac{1}{p}}\left[\left(\max \left\{\left|g^{(n)}\left(x_{1}\right)\right|^{q},\left|g^{(n)}\left(x_{2}\right)\right|^{q}\right\}\right)^{\frac{1}{q}}\right. \\
& \left.+\left(2^{m-1}-1\right)\left(\max \left\{\left|g^{(n)}\left(x_{1}\right)\right|^{q},\left|g^{(n)}\left(x_{2}\right)\right|^{q}\right\}\right)^{\frac{1}{q}}\right] \\
= & \frac{1}{2^{m n} n !}\left(\frac{1}{1+p n}\right)^{\frac{1}{p}}\left(\max \left\{\left|g^{(n)}\left(x_{1}\right)\right|^{q},\left|g^{(n)}\left(x_{2}\right)\right|^{q}\right\}\right)^{\frac{1}{q}}
\end{aligned}
$$

Using (2.3) and (2.21), the result follows.

Theorem 2.6. Suppose that $n$ and $m$ are positive integers, $g: J \subseteq \mathbb{R} \rightarrow \mathbb{R}$ is $n$-time differentiable mapping on $J^{\circ}$ and $x_{1}, x_{2} \in J^{\circ}$ with $x_{1}<x_{2}$. If $g^{(n)} \in L_{1}\left(\left[x_{1}, x_{2}\right]\right)$ and $\left|g^{(n)}\right|^{q}$ is quasi-convex on $\left[x_{1}, x_{2}\right]$, where $q \geq 1$, then

$$
\begin{aligned}
& \mid \int_{x_{1}}^{x_{2}} g(x) d x-\sum_{l=1}^{2^{m-1}} \sum_{k=0}^{n-1}\left[\frac{\left(1+(-1)^{k}\right) 2^{-m(k+1)}}{\left(x_{2}-x_{1}\right)^{-k-1}(k+1) !}\right. \\
& \left.\times g^{(k)}\left(\frac{(2 l-1) x_{1}+\left(2^{m}-2 l+1\right) x_{2}}{2^{m}}\right)\right] \mid \\
& \leq \frac{\left(x_{2}-x_{1}\right)^{n+1}}{2^{m n}(n+1) !}\left(\max \left\{\left|g^{(n)}\left(x_{1}\right)\right|^{q},\left|g^{(n)}\left(x_{2}\right)\right|^{q}\right\}\right)^{\frac{1}{q}}
\end{aligned}
$$

Proof. Using quasi-convexity of $\left|g^{(n)}\right|^{q}$ on $\left[x_{1}, x_{2}\right]$, we get that

$$
\begin{aligned}
& \left|\int_{0}^{1} P_{n, m}(t) g^{(n)}\left(x_{2}+t\left(x_{1}-x_{2}\right)\right) d t\right| \\
& \leq \frac{2}{2^{m n+m}(n+1) !}\left(\max \left\{\left|g^{(n)}\left(x_{1}\right)\right|^{q},\left|g^{(n)}\left(x_{2}\right)\right|^{q}\right\}\right)^{\frac{1}{q}} \\
& +\frac{2}{2^{m n+m}(n+1) !}\left(2^{m-1}-1\right)\left(\max \left\{\left|g^{(n)}\left(x_{1}\right)\right|^{q},\left|g^{(n)}\left(x_{2}\right)\right|^{q}\right\}\right)^{\frac{1}{q}} \\
& =\frac{1}{2^{m n}(n+1) !}\left(\max \left\{\left|g^{(n)}\left(x_{1}\right)\right|^{q},\left|g^{(n)}\left(x_{2}\right)\right|^{q}\right\}\right)^{\frac{1}{q}} .
\end{aligned}
$$

Using (2.3) and (2.23), the result follows.

\section{Some Applications}

We start this section with an application to approximation of an integral. Recall that a partition $D$ of a finite interval $[c, d], c<d$, is a finite sequence of numbers $c=c_{0}<c_{1}<\cdots<c_{n}=d$.

Proposition 3.1. Suppose that $n$ and $m$ are positive integers, $g: J \subseteq \mathbb{R} \rightarrow \mathbb{R}$ is n-time differentiable mapping on $J^{\circ}$ and $c, d \in J^{\circ}$ with $c<d$. Let $D: c=c_{0}<c_{1}<\cdots<c_{n}=d$ be a partition of $[c, d]$. If 
$g^{(n)} \in L_{1}([c, d])$ and $\left|g^{(n)}\right|$ is convex on $[c, d]$, then

$$
\int_{c}^{d} g(x) d x=A(g, D)+E(g, D)
$$

where

$$
\begin{aligned}
& \quad A(g, D) \\
& =\sum_{j=0}^{n-1} \sum_{l=1}^{2^{m-1}} \sum_{k=0}^{n-1}\left[\frac{\left(1+(-1)^{k}\right) 2^{-m(k+1)}}{\left(c_{j+1}-c_{j}\right)^{-k-1}(k+1) !}\right. \\
& \left.\quad \times g^{(k)}\left(\frac{(2 l-1) c_{j}+\left(2^{m}-2 l+1\right) c_{j+1}}{2^{m}}\right)\right],
\end{aligned}
$$

and

$$
\begin{aligned}
& |E(g, D)| \\
\leq & \frac{1}{2^{m n+1}(n+1) !} \sum_{j=0}^{n-1}\left(c_{j+1}-c_{j}\right)^{n+1}\left(\left|g^{(n)}\left(c_{j}\right)\right|+\left|g^{(n)}\left(c_{j+1}\right)\right|\right) .
\end{aligned}
$$

Proof. For each $j=0,1, \cdots, n-1$, applying Theorem 2.1 over the interval $\left[c_{j}, c_{j+1}\right]$,we have

$$
\begin{aligned}
& \mid \int_{c_{j}}^{c_{j+1}} g(x) d x-\sum_{l=1}^{2^{m-1}} \sum_{k=0}^{n-1}\left[\frac{\left(1+(-1)^{k}\right) 2^{-m(k+1)}}{\left(c_{j+1}-c_{j}\right)^{-k-1}(k+1) !}\right. \\
& \left.\times g^{(k)}\left(\frac{(2 l-1) c_{j}+\left(2^{m}-2 l+1\right) c_{j+1}}{2^{m}}\right)\right] \mid \\
& \leq \frac{\left(c_{j+1}-c_{j}\right)^{n+1}}{2^{m n}(n+1) !}\left[\frac{\left|g^{(n)}\left(c_{j}\right)\right|+\left|g^{(n)}\left(c_{j+1}\right)\right|}{2}\right] .
\end{aligned}
$$

Note that

$$
\begin{aligned}
E(g, D) & =\int_{c}^{d} g(x) d x-A(g, D) \\
& =\sum_{j=0}^{n-1}\left(\left[\int_{c_{j}}^{c_{j+1}} g(x) d x-\sum_{l=1}^{2^{m-1}} \sum_{k=0}^{n-1} \frac{\left(1+(-1)^{k}\right) 2^{-m(k+1)}}{\left(c_{j+1}-c_{j}\right)^{-k-1}(k+1) !}\right.\right. \\
& \left.\left.\times g^{(k)}\left(\frac{(2 l-1) c_{j}+\left(2^{m}-2 l+1\right) c_{j+1}}{2^{m}}\right)\right]\right) .
\end{aligned}
$$

Using the triangle inequality and Inequality 3.4 , we get that

$$
|E(g, D)| \leq \frac{1}{2^{m n+1}(n+1) !} \sum_{j=0}^{n-1}\left(c_{j+1}-c_{j}\right)^{n+1}\left(\left|g^{(n)}\left(c_{j}\right)\right|+\left|g^{(n)}\left(c_{j+1}\right)\right|\right) .
$$

The second application will be devoted to random variables. 
Proposition 3.2. Let $X$ be a random variable taking its values in the finite interval $[c, d]$, where $0<c<d$, with a probability density function $g:[c, d] \rightarrow[0,1]$. If $g$ is $n$-time differentiable, $g^{(n)} \in L_{1}([c, d])$ and $\left|g^{(n)}\right|$ is convex on $[c, d]$, then for any positive integer $m$,

$$
\begin{aligned}
& \mid E(X)-\left[d-\sum_{l=1}^{2^{m-1}} \sum_{k=1}^{n}\left(\frac{\left(1+(-1)^{k}\right) 2^{-m(k+1)}}{(d-c)^{-k-1}(k+1) !}\right.\right. \\
& \left.\quad \times g^{(k-1)}\left(\frac{(2 l-1) c+\left(2^{m}-2 l+1\right) d}{2^{m}}\right)\right) \\
& \left.\quad-\frac{2^{-m+1}}{(d-c)^{-1}} \sum_{l=1}^{2^{m-1}} \operatorname{Pr}\left(X \leq \frac{(2 l-1) c+\left(2^{m}-2 l+1\right) d}{2^{m}}\right)\right] \mid \\
& \leq \frac{(d-c)^{n+2}}{2^{m(n+1)}(n+2) !}\left[\frac{\left|g^{(n)}(c)\right|+\left|g^{(n)}(d)\right|}{2}\right],
\end{aligned}
$$

where $E(X)$ is the expectation of $X$.

Proof. Let $G(x)=\int_{c}^{x} g(t) d t$ for $x \in[c, d]$. Using integration by parts and the facts that $G^{\prime}=g$ and $G(d)=1$, we get that

$$
E(X)=d-\int_{c}^{d} G(t) d t
$$

Since $G^{(k+1)}=g^{(k)}, 0 \leq k \leq n$, applying Theorem 2.1 on $G$, we have

$$
\begin{aligned}
& \mid \int_{c}^{d} G(t) d t-\sum_{l=1}^{2^{m-1}} \sum_{k=1}^{n}\left[\frac{\left(1+(-1)^{k}\right) 2^{-m(k+1)}}{(d-c)^{-k-1}(k+1) !}\right. \\
& \left.\quad \times g^{(k-1)}\left(\frac{(2 l-1) c+\left(2^{m}-2 l+1\right) d}{2^{m}}\right)\right] \\
& \quad-\frac{2^{-m+1}}{(d-c)^{-1}} \sum_{l=1}^{2^{m-1}} \operatorname{Pr}\left(X \leq \frac{(2 l-1) c+\left(2^{m}-2 l+1\right) d}{2^{m}}\right) \mid \\
& \leq \frac{(d-c)^{n+2}}{2^{m(n+1)}(n+2) !}\left[\frac{\left|g^{(n)}(c)\right|+\left|g^{(n)}(d)\right|}{2}\right] .
\end{aligned}
$$

Thus,

$$
\begin{aligned}
& \mid E(X)-\left[d-\sum_{l=1}^{2^{m-1}} \sum_{k=1}^{n}\left(\frac{\left(1+(-1)^{k}\right) 2^{-m(k+1)}}{(d-c)^{-k-1}(k+1) !}\right.\right. \\
& \left.\quad \times g^{(k-1)}\left(\frac{(2 l-1) c+\left(2^{m}-2 l+1\right) d}{2^{m}}\right)\right) \\
& \left.\quad-\frac{2^{-m+1}}{(d-c)^{-1}} \sum_{l=1}^{2^{m-1}} \operatorname{Pr}\left(X \leq \frac{(2 l-1) c+\left(2^{m}-2 l+1\right) d}{2^{m}}\right)\right] \mid \\
& \leq \frac{(d-c)^{n+2}}{2^{m(n+1)}(n+2) !}\left[\frac{\left|g^{(n)}(c)\right|+\left|g^{(n)}(d)\right|}{2}\right] .
\end{aligned}
$$


Remark 3.1. In Proposition 3.2, if $m=1$ then Identity 3.5 reduces to

$$
\begin{gathered}
\mid E(X)-d+(d-c) \operatorname{Pr}\left(X \leq \frac{c+d}{2}\right) \\
+\sum_{k=1}^{n} \frac{\left(1+(-1)^{k}\right) 2^{-(k+1)}}{(d-c)^{-k-1}(k+1) !} g^{(k-1)}\left(\frac{c+d}{2}\right) \mid \\
\leq \frac{(d-c)^{n+2}}{2^{(n+1)}(n+2) !}\left[\frac{\left|g^{(n)}(c)\right|+\left|g^{(n)}(d)\right|}{2}\right] .
\end{gathered}
$$

Conflicts of Interest: The author(s) declare that there are no conflicts of interest regarding the publication of this paper.

\section{REFERENCES}

[1] P. Cerone, S.S. Dragomir, J. Roumeliotis and J. Sunde, A new generalization of the trapezoid formula for n-time differentiable mappings and applications, Demonstr. Math. 33 (4) (2000), 719-736.

[2] X. L. Cheng, Improvement of some Ostrowski-Grüss type inequalities, Computers Math. Appl. 42 (2001), 109-114.

[3] L. Chun and F. Qi, Integral inequalities of Hermite-Hadamard type for functions whose third derivatives are convex, J. Inequal. Appl. 2013 (2013), Art. ID 451.

[4] S. S. Dragomir and S. Wang, An inequality of Ostrowski-Grüss type and its applications to the estimation of error bounds for some special means and for home numerical quadrature rules, Computers Math. Appl. 33 (11) (1997), 15-20.

[5] D. Y. Hwang, Some Inequalities for $n$-time Differentiable Mappings and Applications, Kyung. Math. J. 43 (2003), 335-343.

[6] J. L. W. V. Jensen, On konvexe funktioner og uligheder mel lem middlvaerdier, Nyt. Tidsskr. Math. B. 16 (1905), $49-69$.

[7] A. I. Kechriniotis and Y. A. Theodorou, New integral inequalities for $n$-time differentiable functions with applications for pdfs, Appl. Math. Sci. 2 (8) (2008), 353-362.

[8] A. Ostrowski, Uber die Absolutabweichung einer differentierbaren Funktion von ihren Integralmittelwert, Comment. Math. Helv., 10 (1938), 226-227.

[9] M. E. Ozdemir and C. Yıldız, A new generalization of the midpoint formula for $n$-time differentiable mappings which are convex, arXiv:1404.5128v1, 2014.

[10] B.G. Pachpatte, New inequalities of Ostrowski and Trapezoid type for $n$-time differentiable functions, Bull. Korean Math. Soc. 41 (4) (2004), 633-639.

[11] J. E. Pečarič, F. Proschan, Y.L. Tong, Convex Function, Partial Ordering and Statistical Applications, Academic Press, New York, 1991.

[12] S. H. Wang and F. Qi, Inequalities of Hermite-Hadamard type for convex functions which are $n$-times differentiable, Math. Inequal. Appl. 16 (4) (2013), 1269-1278. 